\title{
Novel Usher syndrome pathogenic variants identified in cases with hearing and vision loss
}

Justin A. Pater ${ }^{1}$, Jane Green ${ }^{1}$, Darren D. O'Rielly ${ }^{3}$, Anne Griffin ${ }^{1}$, Jessica Squires ${ }^{1}$, Taylor Burt ${ }^{1}$, Sara Fernandez², Bridget Fernandez ${ }^{1,2}$, Jim Houston $^{1}$, Jiayi Zhou' ${ }^{1}$, Nicole M. Roslin ${ }^{4}$ and Terry-Lynn Young ${ }^{1,3^{*}}$ (D)

\begin{abstract}
Background: Usher syndrome, the most common form of inherited deaf-blindness, is unlike many other forms of syndromic hereditary hearing loss in that the extra aural clinical manifestations are also detrimental to communication. Usher syndrome patients with early onset deafness also experience vision loss due to progressive retinitis pigmentosa that can lead to legal blindness in their third or fourth decade.

Methods: Using a multi-omic approach, we identified three novel pathogenic variants in two Usher syndrome genes (USH2A and ADGRV1) in cases initially referred for isolated vision or hearing loss.

Results: In a multiplex hearing loss family, two affected sisters, the product of a second cousin union, are homozygous for a novel nonsense pathogenic variant in ADGRV1 (c.17062C > T, p.Arg5688*), predicted to create a premature stop codon near the N-terminus of ADGRV1. Ophthalmological examination of the sisters confirmed typical retinitis pigmentosa and prompted a corrected Usher syndrome diagnosis. In an unrelated clinical case, a child with hearing loss tested positive for two novel USH2A splicing variants (c.5777-1G > A, p. Glu1926_Ala1952del and c.10388-2A > G, p.Asp3463Alafs*6) and RNA studies confirmed that both pathogenic variants cause splicing errors. Interestingly, these same USH2A variants are also identified in another family with vision loss where subsequent clinical follow-up confirmed pre-existing hearing loss since early childhood, eventually resulting in a reassigned diagnosis of Usher syndrome.
\end{abstract}

Conclusion: These findings provide empirical evidence to increase Usher syndrome surveillance of at-risk children. Given that novel antisense oligonucleotide therapies have been shown to rescue retinal degeneration caused by USH2A splicing pathogenic variants, these solved USH2A patients may now be eligible to be enrolled in therapeutic trials.

Keywords: Syndromic hearing loss, Usher syndrome, RNA splicing, Knowledge translation, Whole exome sequencing, Genetic isolate

\section{Background}

Approximately $30 \%$ of inherited hearing loss is syndromic and is classically characterized by overt clinical features, such as distinctive craniofacial and eye abnormalities, and joint problems as in Stickler syndrome [1]

\footnotetext{
* Correspondence: tlyoung@mun.ca

${ }^{1}$ Craig L. Dobbin Research Centre, Discipline of Genetics, Faculty of Medicine, Memorial University, St. John's, Newfoundland \& Labrador AIB 3V6, Canada ${ }^{3}$ Molecular Diagnostic Laboratory, Eastern Health, Craig L. Dobbin Genetics Research Centre, Faculty of Medicine, Memorial University, 300 Prince Phillip Drive, St. John's, Newfoundland and Labrador A1B 3V6, Canada Full list of author information is available at the end of the article
}

(MIM: 108300). However, syndromic forms of hearing loss such as Usher syndrome (USH), present more insidiously, often resulting in delayed or misdiagnosis. USH is an autosomal recessive condition characterized by bilateral sensorineural hearing loss with or without vestibular dysfunction, and progressive retinitis pigmentosa (RP) [2-4]. Most children with USH are born with congenital hearing loss; however, progressive RP may present in the second decade, making diagnosis difficult due to the subtle changes in visual function over time [5]. Historically, USH was considered an extremely rare

(c) The Author(s). 2019 Open Access This article is distributed under the terms of the Creative Commons Attribution 4.0 International License (http://creativecommons.org/licenses/by/4.0/), which permits unrestricted use, distribution, and reproduction in any medium, provided you give appropriate credit to the original author(s) and the source, provide a link to the Creative Commons license, and indicate if changes were made. The Creative Commons Public Domain Dedication waiver (http://creativecommons.org/publicdomain/zero/1.0/) applies to the data made available in this article, unless otherwise stated. 
disorder with a frequency of 1 in 25,000 [6]; however, a recent study suggests a higher prevalence of 1 in 6000 individuals in the European (Non-Finnish) population [3].

USH is an extremely deleterious disorder and is the most common cause of inherited deaf-blindness [3]. So far, 13 USH genes have been identified which adversely affect the development of sensory hair cells within the inner ear and of photoreceptors in the eye [5]. The most common subtype, USH type 2A (USH2A), accounts for two-thirds of all cases. Many USH $2 \mathrm{~A}$ pathogenic variants cause splicing defects such as exon skipping and the creation or destruction of canonical acceptor and donor splice sites [5]. Novel therapies that target USH2A show great promise as retinal degeneration in USH2A patients can be rescued using antisense oligonucleotide-based therapy targeting cryptic splicing variants [7]. Additionally, antioxidant-based therapies have also shown great promise in preventing cone degeneration in USH1 mice, which is linked to oxidative stress [8]. Oxidative stress has well-established roles in many retinal dystrophies, where polymorphisms in GLO1 may explain RP susceptibility and clinical heterogeneity [9]. Enrollment of patients in therapeutic trials requires a molecular diagnosis which can be challenging in the clinical setting. A comprehensive approach that includes linkage analysis, exome sequencing and functional analysis is often required, especially for novel splicing variants $[10,11]$. Herein, we report three novel USH pathogenic variants in $U S H 2 A$ or $A D V R G 1$ identified in cases of vision and hearing loss using a comprehensive multi-omic approach.

\section{Methods}

\section{Study participants and clinical evaluations}

The study involved three families from the Newfoundland population, including two multiplex families. Clinical evaluations included air conduction thresholds using pure-tone audiometry, noting the audiogram configuration, severity, onset and progression. Vision was assessed with ocular examination, visual acuity and visual field testing, electroretinography (ERG) and fluorescein angiography of the retina.

For Family R2100, hearing loss (HL) is present in three sibships with varying audioprofiles, including two sisters who are the product of a consanguineous union (Fig. 1a). The proband (PID V-2) diagnosed with hearing loss at 3 years, presents by age 7 with a mild to moderate bilateral sensorineural HL, and her younger sister (PID V-3) was diagnosed at age 3 with a similar audioprofile (Fig. 1b).

We also recruited a case from our local medical genetics' clinic (Family R4110) of a child diagnosed at 3 months (following newborn hearing screening), who presents by age 3 with mild to moderate bilateral sensorineural HL (Fig. 1 c, d). In another multiplex family
(R0723), the proband and his brothers (PIDs II-5, II-3 and II-6, respectively) were first diagnosed with RP in mid 5th decade when their central vision decreased to the point that they met criteria for legally recognized blindness (Fig. 1e). They reported reduced night vision since the mid-second decade, and hearing loss since young childhood. The proband had been fitted for hearing aids for moderate to severe hearing loss. In R0273, the proband and his brothers (PIDs II-5, II-3 and II-6 respectively) had reported experiencing reduced night vision since their mid-third decade and were all diagnosed with RP in the mid-fifth decade when their central vision decreased. Throughout the course of ongoing clinical assessment, it was noted that the proband was fitted for hearing aids due to a moderate to severe $\mathrm{HL}$ and although the age of onset was unknown, he had HL at a young age. Two nieces with early hearing loss were also diagnosed with RP on follow-up, which prompted targeted genetic testing for known USH genes (Fig. 1d).

\section{Gene panels}

In the case of family R0723, a targeted gene panel for 13 USH genes (CEI Molecular Diagnostic Laboratory, Portland, OR, USA) was offered through a research study on hereditary vision loss. In the clinical case of the child who failed newborn hearing screening (Family R4110), the family was offered targeted screening (158 syndromic and non-syndromic hearing loss genes, Blueprint Genetics, Comprehensive Hearing Loss and Deafness Panel, version 1, San Francisco, CA, USA). To validate variants of interest and check for co-segregation with RP and/or HL trait in the families, genomic DNA was amplified using custom primers and sequenced in both directions using standard touchdown PCR protocols $(\mathrm{ABI}$ PRISM 3500XL DNA Analyzer; Applied Biosystems, Foster City, CA, USA). Sequence traces were analyzed using Mutation Surveyor Software (version 5.00, SoftGenetics LLC State College, PA 16803).

\section{Linkage analysis and whole exome sequencing in hearing loss family R2100}

We initially screened the proband of Family 2100 for deafness alleles previously identified in the NL population and submitted representative audiograms to Audiogene, a program comparing these to average audiograms of 34 deafness loci [12]. As this targeted approach failed to solve Family 2100, a traditional linkage study was done. For the linkage analysis, we selected three affected and two unaffected relatives (PID V-2, V-3, III-9, and IV-3, IV-4 respectively) (Fig. 1a) and genotyped 17,407 polymorphic markers with the Illumina Human610-Quad chip. Multipoint linkage analysis (Merlin v1.1.2) [13] was performed under a recessive model with a disease allele frequency of 


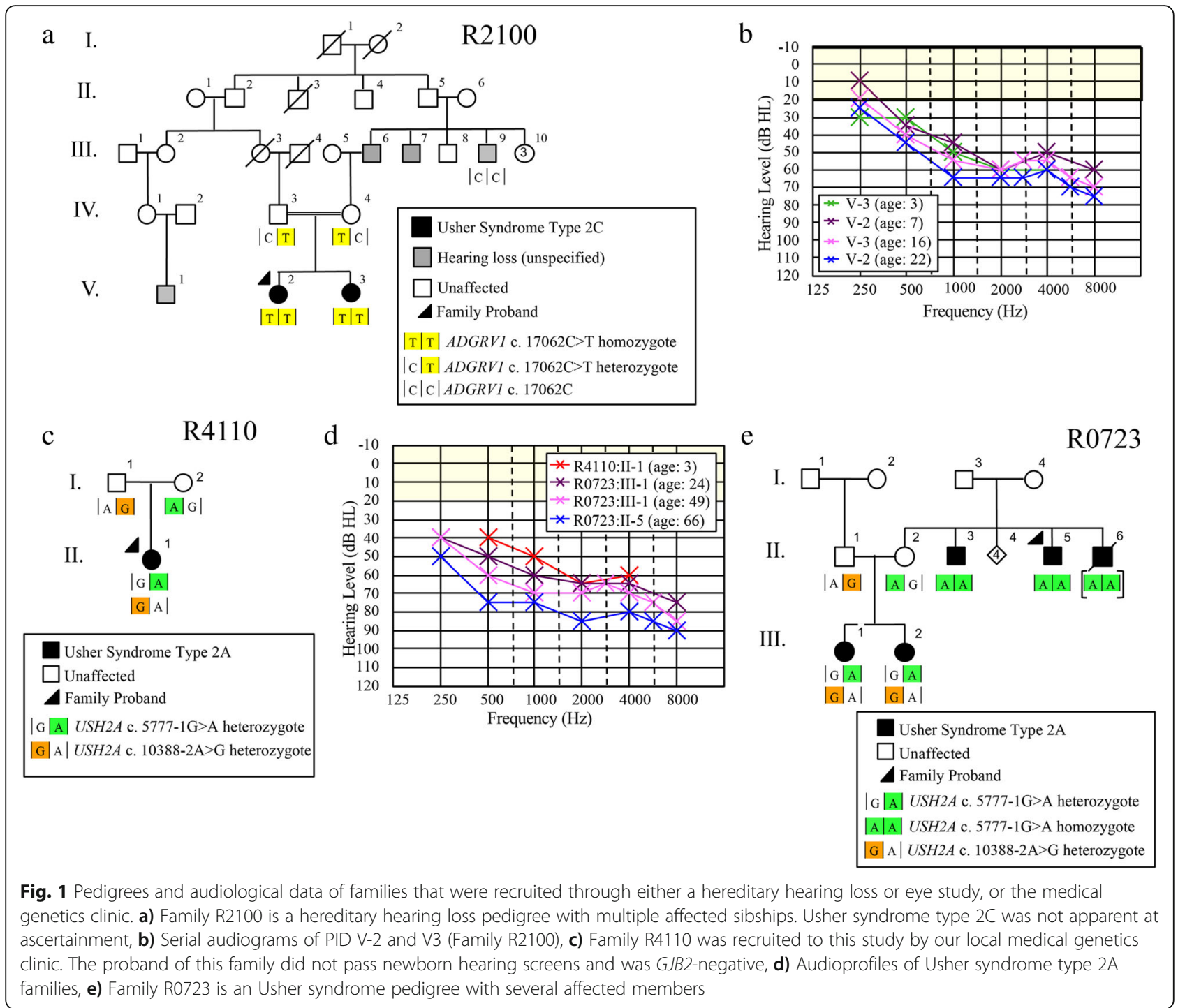

0.07 and a penetrance of $99 \%$. In order to screen candidate genes within linked regions, whole exome sequencing was carried out on 5 family members (two affected offspring and their parents: PID V-2, V-3, IV-3, IV-4 respectively) using the Ion Torrent AmpliSeq RDY Exome Kit (Life Technologies, Cat. \#A27193). Purified libraries were loaded onto an Ion Proton PI v3 chip and sequenced with the Ion Torrent Proton. Only rare variants $(\mathrm{MAF}<1 \%)$ that mapped to linked regions, had a depth of coverage $>20 \mathrm{X}$ and were of medium to high impact were validated by Sanger sequencing and selected for cascade screening. Population frequencies were determined using 124 ethnically-matched controls.

\section{Splice variant in silico analysis}

For variants of interest that reside within canonical +/1 or 2 splice sites, we conducted in silico analyses using
Alamut Visual (Interactive Biosoftware Inc., Rouen, France), a program that provides a splicing alteration report by linking to the MaxEnt, NNSPPLICE, SplicSiteFinder, and GeneSplicer algorithms.

\section{RNA-CDNA validation of splice variants}

In order to experimentally validate splicing predictions, we extracted total RNA from B-cell lymphocytes using standard TRIzol-based methods (Thermo-fisher, Cat. \#15596026) and prepared cDNA libraries with the Superscript III First Strand Synthesis System (Thermo-fisher, Cat. \#18080093). RT-PCR was carried out with primers that spanned candidate splicing regions, followed by TOPO TA-Cloning Kit for Sequencing with One Shot TOP10 Chemically Competent E. coli (Invitrogen, \#K457540) according to the manufacturer's protocol. RT-PCR products were Sanger 
sequenced and then analyzed using Mutation Surveyor Software (version 5.00, SoftGenetics LLC State College, PA 16803).

\section{Results}

ADGRV1 c.17062C > T genotype/phenotype analyses

In the step-wise analysis of hearing loss Family 2100, the proband (PID V-2; Fig. 1a) screened negative for all hearing loss variants previously identified in the NL population. Genome-wide linkage analysis (assuming autosomal recessive inheritance) yielded positive LOD scores suggestive of linkage for 8 genomic regions and the theoretical maximum LOD (1.68) for regions on chromosomes 3, 4, 5, 6 and 15 (Table 1). Subsequently, exome sequencing identified 278 variants that were shared between the proband (PID V-2) and her affected sister (PID V-3). Of these, only eight variants remained after filtering for rare variants $(\mathrm{MAF}<1 \%)$ of medium to high impact that mapped to linked regions and had a depth of coverage $>20 \mathrm{X}$ (Table 2). Seven of these variants were shown to be false positive INDELs (did not validate with Sanger sequencing) or did not reside within genes associated with syndromic or non-syndromic HL [14]. The remaining candidate (ADGRV1 c.17062C $>\mathrm{T}$; p.Arg5688*) is a nonsense variant associated with USH $2 \mathrm{C}$ (Fig. 2a). Co-segregation analysis confirmed that the affected sisters were homozygous for ADGRV1 c. $17062 \mathrm{C}>\mathrm{T}$ and their parents were unaffected carriers. The only other available affected relative for cascade sequencing was a maternal uncle (PID III-9; Fig. 1a) who was wild-type (two normal copies) and subsequently confirmed to have acquired his hearing loss after a serious diving accident. The nonsense ADGRV1 variant is predicted to create a premature stop codon nearing the $\mathrm{N}$-terminus of ADGRV1, preventing the translation of all 7 transmembrane domains. Furthermore, the ADGRV1 c. $17062 \mathrm{C}>\mathrm{T}$ variant is absent in the population controls and has a single heterozygous entry in ExAC browser from the European (Non-Finnish) population. According to ACMG guidelines, the ADGRV1 nonsense variant should be classified as pathogenic as it meets the following criteria: PVS1, PM2, PM3, PP1, PP3.

At the start of this study, we were aware of hearing loss (HL) in three sibships with varying audioprofiles, including two sisters who are the product of a consanguineous union. On the basis of the pending molecular diagnosis of Usher syndrome and the serious prognosis, the clinic contacted the sisters in order to request a visual examination (PID V-2 and V-3; Fig. 1a). The sisters are now in their late third and early fourth decade. Both women report impaired vision for some years. Ophthalmology reports on both sisters indicated definite features of RP. Further testing of PID V-3 identified bone spicule pigmentation of the retina (Fig. $2 \mathrm{~b}$ ) and a significant reduction in peripheral visual acuity (Fig. 2c), which are consistent with "typical RP". These findings prompted the clinic to counsel the women regarding their new diagnosis of USH2C.

\section{USH2A c.5777-1G > A and c.10388-2A > G genotype/ phenotype analyses}

The comprehensive gene panel that was offered to the clinical case of the 3-year-old child diagnosed with isolated hearing loss at 3 months (Family 4110; Fig. 1c) identified two novel USH2A splicing variants: c.5777-1G > A (Fig. 2d) and c.10388-2A > G (Fig. 2e). Cascade sequencing confirmed the maternal contribution as c.5777-1G $>\mathrm{A}$ and the paternal contribution as c.10388-2A $>\mathrm{G}$, and verified these novel variants reside in trans. However, given this child's young age and the novelty (variants of unknown significance) of the $U S H 2 A$ variants, the genetic testing results are of limited value.

Fortuitously, the targeted USH gene panel offered to Family R0723 identified these same USH $2 A$ splicing variants. The proband (PID II-5) and his brother (PID II-3) are homozygous for UHS2A c.5777-1G > A, their nieces (PIDs III-1 and III-2) are compound heterozygotes (c.5777-1G > A; c.10388-2A > G; Fig. 1e). Even though the deceased brother (PID II-6) was not

Table 1 R2100 Multipoint Linkage Analysis Result

\begin{tabular}{|c|c|c|c|c|c|c|}
\hline \multirow[b]{2}{*}{ Chromosome } & \multirow[b]{2}{*}{ LOD } & \multicolumn{2}{|l|}{ Start } & \multicolumn{2}{|l|}{ End } & \multirow[b]{2}{*}{ Size (Mb) } \\
\hline & & dbSNP & Position & $\mathrm{dbSNP}$ & Position & \\
\hline 1 & 0.58 & rs3933251 & $64,095,165$ & rs591540 & $71,866,132$ & 7.8 \\
\hline 3 & 1.68 & rs1400207 & $2,825,953$ & rs13084851 & $4,115,819$ & 1.3 \\
\hline 4 & 1.68 & rs7671597 & $41,665,756$ & rs2035906 & $45,815,310$ & 4.1 \\
\hline 5 & 1.68 & rs12110158 & $78,378,722$ & rs257239 & $97,997,738$ & 19.6 \\
\hline 6 & 1.67 & rs1322633 & $125,082,133$ & rs1490388 & $126,514,509$ & 1.4 \\
\hline 11 & 1.64 & rs1320211 & $15,301,410$ & rs10833818 & $22,795,026$ & 7.5 \\
\hline 15 & 1.68 & rs937302 & $33,627,848$ & rs11070349 & $41,792,819$ & 8.2 \\
\hline 20 & 1.41 & rs237417 & $5,770,142$ & rs4140471 & $7,226,740$ & 1.5 \\
\hline
\end{tabular}


Table 2 Eight variants identified by whole exome sequencing

\begin{tabular}{|c|c|c|c|c|c|c|c|}
\hline Chr & Gene & REF & ALT & SNP Position & $\mathrm{dbSNP}$ & EXAC MAF (\%) & Protein Effect \\
\hline 6 & HEY2 & A & $A C, C$ & $126,080,841$ & None & None & INDEL \\
\hline 3 & WDR48 & GTTTG & GTT, GTTG & $39,136,139$ & None & None & INDEL \\
\hline 3 & RPL 14 & A & ACTGCTG & $40,503,520$ & None & None & INDEL \\
\hline 5 & MTX3 & G & C & $79,281,458$ & None & None & Missense \\
\hline 5 & ADGRV1 & C & $\mathrm{T}$ & $90,144,496$ & rs747622607 & 0.003235 & Nonsense \\
\hline 5 & SPATA9 & A & G & $95,011,189$ & rs55796768 & 0.958466 & Missense \\
\hline 5 & ERAP1 & C & G & $96,139,250$ & None & None & Missense \\
\hline 15 & GOLGA8A & C & $\mathrm{T}$ & $34,673,722$ & rs765222922 & None & Missense \\
\hline
\end{tabular}

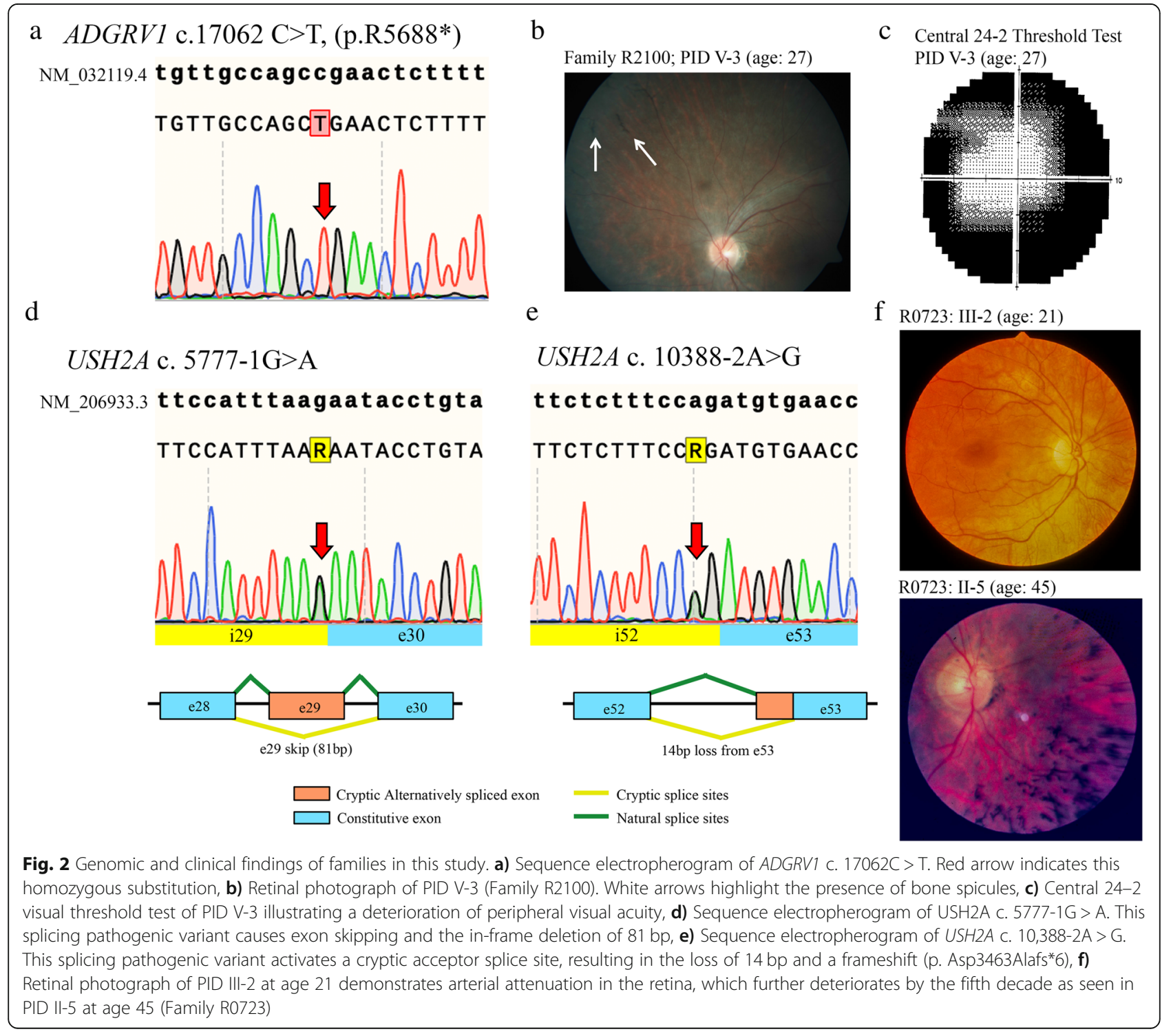


available for genetic testing, he is likely a $U S H 2 A$ c.5777-1G > A homozygote, given the strong family history of RP. Retrospective audiological data on the proband's niece, PID III-1, from mid-third decade to mid-fifth decade show a stable hearing loss according to GenDeaf guidelines (Fig. 1d) [15]. The proband (PID II-5) has moderate to severe hearing loss in his seventh decade, not significantly worse than his younger niece (PID III-1) in her late fifth decade (Fig. 1d). His other niece, PID III-2, reveals a similar clinical phenotype (data not shown). With respect to RP, the proband (PID II-5) and his two brothers (PID II-3 and II-6) reported decreased night vision by their late 20s (Fig. 1e); however, RP as seen in retinal photographs of the proband was not diagnosed in the brothers until their late 40's (Fig. 2f). Following the diagnosis of RP in the uncles, their nieces who had documented hearing loss were closely monitored, and reduced visual fields noted at age 14 in PID III-2, indicating the first symptoms of RP. Abnormal dark adaptation and ERG responses were recorded in both nieces in the third decade and retinal photographs of PID III-2 illustrate arterial attenuation, a characteristic sign of early RP (Fig. 2f).

\section{USH2A c.5777-1G > A and c.10388-2A > G experimental validation of splicing effects}

Cascade sequencing revealed that both $U S H 2 A$ c.5777-1G > A and USH2A c.10388-2A > G co-segregate with disease in families R0723 and R4110. In silico analyses using Alamut Visual suite of algorithms predicted that both variants cause exon skipping (MaxEnt: $-100.0 \%$, NNSPLICE: $-100.0 \%$ and SSF: - 100.0\%). Using patient-derived cells, Sanger sequencing of cDNA confirmed that USH2A c.5777-1G > A causes the skipping of exon 29 leading to an in-frame deletion (p. Glu1926_Ala1952del) in an affected individual (PID III-2) compared with a control sample (Fig. 2d; Fig. 3a). The sequencing

a

USH2A c.5777-1G (wild-type allele, NM_206933.3)

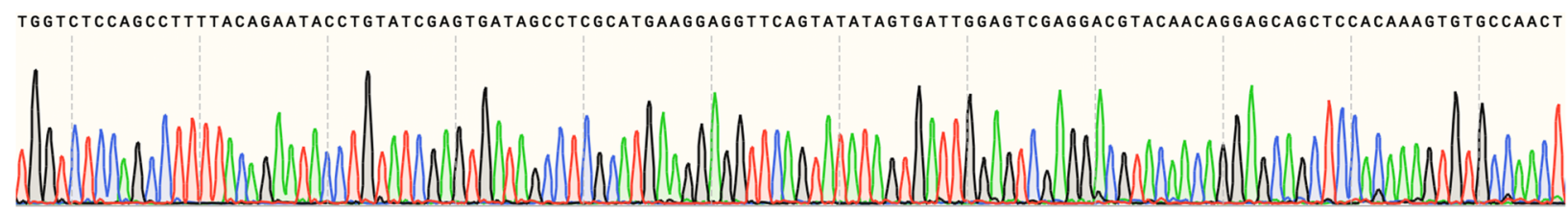

USH2A c.5777-1G>A (mutant allele)

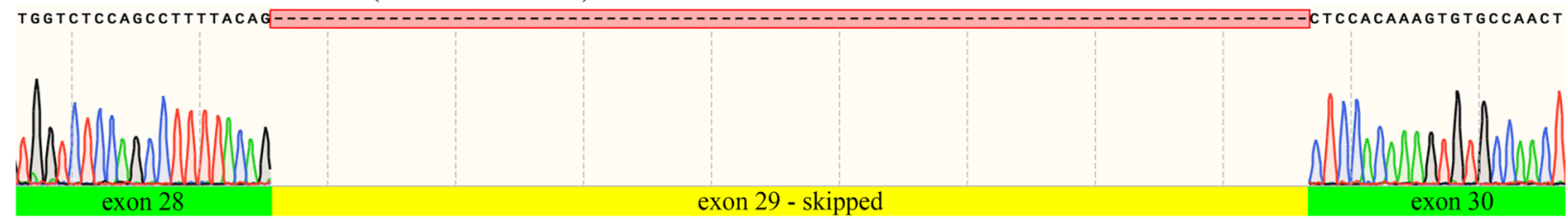

$\mathrm{b}$

USH2A c.10388-2A (wild-type allele, NM_206933.3)

ATCTGCCGAAGAAACCATTCATACAGGGAGTGTAAACACGTACTCTTACACA $\overline{G A T G T G A A C C T C A A G C C C T A C A T G A C A T A T G A G T A C A G G A T T T C T G C C T G G A A C A G C T A T G G G C G A G G A}$

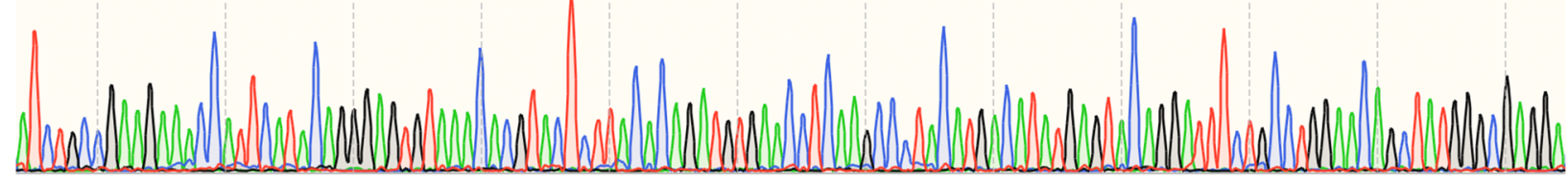

USH2A c. $10388-2 \mathrm{~A}>\mathrm{G}$ (mutant allele)

ATCTGCCGAAGAAACCATTCATACAGgGGTGTAAACACGTACTCTTACACAG -

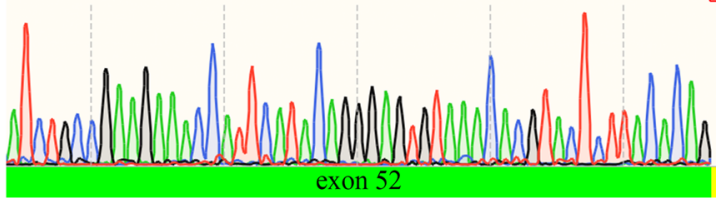

$14 \mathrm{bp}$ deletion

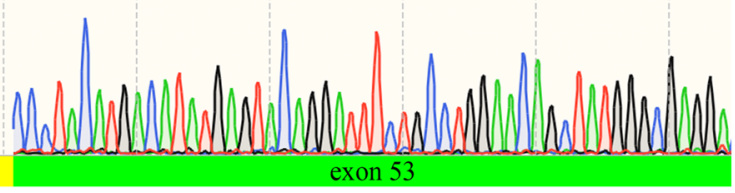

Fig. 3 Sequence electropherograms of USH2A c. 5777-1G > A and 10,388-2A > G RT-PCR products. a) USH2A c. 5777-1G > A causes exon skipping and the in-frame deletion of 81 bp, b) USH2A 10,388-2A > G activates a cryptic acceptor splice site, resulting in the loss of 14 bp and a frameshift (p. Asp3463Alafs*6) 
of patient cDNA also determined that USH2A c.10388-2A > G activates a cryptic acceptor site $14 \mathrm{bps}$ downstream of the canonical splice site (Fig. 2e; Fig. 3b), resulting in a premature stop codon (p.Asp3463Alafs"6). Based on cascade sequencing within these families and subsequent RNA analysis, USH $2 A$ c.5777-1G $>\mathrm{A}$ and c.10388-2A > G can both be classified as pathogenic variants according to the ACMG guidelines (PVS1, PS3, PM2, PM3, PP1, PP3) [16].

\section{Discussion}

Two novel pathogenic variants in $U S H 2 A$ account for cases recruited or referred as isolated hearing or vision loss in two families in this study. Clinical evidence suggests that the two novel $U S H 2 A$ pathogenic variants result in congenital moderate to severe HL, and RP in the pre/post-pubertal period; findings similar to that of previously reported pathogenic variants in USH2A [17]. Several affected family members present as compound heterozygotes, suggesting that both $U S H 2 A$ c.5777-1G > $\mathrm{A}$ and c.10388-2A $>\mathrm{G}$ pathogenic variants are sufficient to cause USH2A and therefore are USH2A-specific. This finding is consistent with the allelic hierarchy model of USH $2 \mathrm{~A}$ alleles, which suggests that certain alleles are USH2A-specific and others RP-specific, and the presence of at least one RP-specific allele causes isolated RP with normal hearing [18]. Given our clinical case with a young girl who also tested positive for both of these alleles, we are now increasing surveillance for visual symptoms, leading to improved management of USH.

Similarly, we find that two sisters with hereditary hearing loss, the product of a second cousin union, are homozygous for a nonsense pathogenic variant in ADGRV1 (c.17062C > T, p.Arg5688*). Visual examination secondary to molecular analyses confirmed typical RP (late third and fourth decade) in addition to hearing loss (first decade) and prompted a corrected USH diagnosis. This is consistent with previous reports of $A D G R V 1$ pathogenic variants associated with early onset of hearing loss with delayed visual impairment $[19,20]$, most of which are located in the calx- $\beta$ motif [21], and the $A D G R V 1$ c. $17062 C>\mathrm{T}$ lies downstream (3') to this calx $-\beta$ motif. This variant is rare, and to our knowledge, has only been reported once before when it was identified in $1 / 31$ French non-USH2A patients [22]. In addition to causing USH2C, nonsense ADGRV1 pathogenic variants have been shown to cause dominant audiogenic epilepsy [23, 24]. However, the two affected sisters from R2100 whom are homozygous for ADGRV1 c.17062C $>\mathrm{T}$ do not present with audiogenic epilepsy.

Clinically, USH2 should be suspected in patients with bilateral, congenital, sensorineural, mild to severe hearing loss, normal vestibular function, and post-pubertal $\mathrm{RP}$, most often in the second decade [4, 26]. Visual examinations revealed a 'typical RP' phenotype in patients diagnosed with USH2A or USH2C [21]. Likewise, from an audiological standpoint, our data is consistent with previous reports of a stable moderate to severe hearing loss $[19,22,27,28]$. These results indicate that USH2A and USH2C are not readily discerned phenotypically [21]. The USH2A, ADGRV1 and WHRN proteins co-localize at the stereocilia base in developing cochlear hair cells and together form the Ankle-link complex at the base of sensory hair cells and at the periciliary membrane complex of photoreceptors [29-31], so it is not surprising that the USH2A and USH2C phenotypes are indistinguishable.

To determine variant pathogenicity, clinical best-practice guidelines, such as ACMG [16] and EuroGentest [25] are important to follow. For the splicing variants, we used in silico prediction algorithms for preliminary assessment only, and experimentally confirmed the splicing effects using patient-derived B-cell lines. For the nonsense variant, we confirmed that the parents were unaffected carriers and that their affected children received one copy of the novel nonsense ADGRV1 c. $17062 \mathrm{C}>\mathrm{T}$ variant from each of them, establishing that we are detecting two disease alleles in trans and confirming the recessive pattern for Usher syndrome.

In a recent meta-analysis including all of the known genes causing usher syndrome, USH2A (50\%) mutations are the most common with $A D G R V 1$ mutations being less frequent $(5 \%)$ in patients with both visual and hearing impairments [32]. In patients with seemingly isolated sensorineural deafness, $7.5 \%$ had disease-causing mutations in USH genes, and are therefore at high risk of developing RP. In isolated cases of 'hearing loss' or 'vision loss', it is important to screen both USH and RP genes, as an accurate diagnosis of Usher syndrome is essential for patient clinical follow-up, particularly the referral and access to the correct support systems.

\section{Conclusions}

Recognition of syndromic forms of both hearing and vision loss, especially Usher syndrome, is important given the major impact of these types of sensory losses on the acquisition of speech in children and quality of life for adults. In this report, USH was not considered in these cases until genetic testing was performed. Close collaboration between local clinics and molecular genetics researchers was necessary to fully categorize three novel USH variants as pathogenic using ACMG criteria. Accurate molecular diagnosis of patients is essential to provide new opportunities for patients and their families to enroll in therapeutic trials.

\section{Abbreviations}

DNA: Deoxyribonucleic acid; LOD: Logarithm of odds score;

NL: Newfoundland and Labrador; PID: Pedigree identification number; 
RP: Retinitis pigmentosa; RT-PCR: Reverse-transcription polymerase chain reaction; USH: Usher syndrome; USH2A: Usher syndrome type 2A USH2C: Usher syndrome type $2 \mathrm{C}$

\section{Acknowledgements}

We are grateful to the families who participated in this study, to Cindy Penney and Amanda Morgan for technical assistance.

\section{Funding}

This work was supported by operating grants from the Canadian Institutes for Health Research, Genome Canada (Atlantic Medical Genetics and Genomics Initiative) and the Janeway Children's Hospital Foundation; infrastructure grants from the Research and Development Corporation of Newfoundland and Labrador and the Canadian Foundation for Innovation (New Investigator Award no. 9384 and Leaders Opportunity Fund no.13120).

\section{Availability of data and materials}

All data generated or analyzed during this study are included in this published article.

\section{Authors' contributions}

BF, SF, JG, AG, TB contributed patients/analyzed clinical data; NR linkage analysis, JAP, JH; molecular studies, JZ, JAP, JS, DDO bioinformatics, JP, JH, JG, DDO TLY wrote/edited the manuscript, TLY, JG directed the study. All authors have read and approved the manuscript.

\section{Ethics approval and consent to participate}

Informed consent, family history and permission to access audiology (protocol \#01.186) and ophthalmology (protocol \#06.157) records were obtained from all participants per approved institutional review board (Human Research Ethics Board, St. John's, NL, Canada). Parental consent was obtained in the case of minors.

\section{Consent for publication}

Not applicable.

\section{Competing interests}

The authors declare that they have no competing interests.

\section{Publisher's Note}

Springer Nature remains neutral with regard to jurisdictional claims in published maps and institutional affiliations.

\section{Author details}

${ }^{1}$ Craig L. Dobbin Research Centre, Discipline of Genetics, Faculty of Medicine, Memorial University, St. John's, Newfoundland \& Labrador AlB 3V6, Canada. ${ }^{2}$ Provincial Medical Genetics, Craig L. Dobbin Research Centre, Eastern Health, 300 Prince Phillip Drive, St. John's, Newfoundland and Labrador A1B 3V6, Canada. ${ }^{3}$ Molecular Diagnostic Laboratory, Eastern Health, Craig L. Dobbin Genetics Research Centre, Faculty of Medicine, Memorial University, 300 Prince Phillip Drive, St. John's, Newfoundland and Labrador A1B 3V6, Canada. ${ }^{4}$ The Centre for Applied Genomics, The Hospital for Sick Children, 686 Bay Street, Toronto, Ontario M5G 0A4, Canada.

Received: 13 October 2018 Accepted: 1 March 2019

Published online: 02 May 2019

\section{References}

1. Baker S, Booth C, Fillman C, Shapiro M, Blair MP, Hyland JC, Ala-Kokko L: A loss of function mutation in the COL9A2 gene causes autosomal recessive Stickler syndrome. Am J Med Genet A 2011, 155A(7):1668-1672.

2. Keats BJ, Corey DP. The usher syndromes. American journal of medical genetics. 1999:89(3):158-66.

3. Kimberling WJ, Hildebrand MS, Shearer AE, Jensen ML, Halder JA, Trzupek K Cohn ES, Weleber RG, Stone EM, Smith RJ. Frequency of Usher syndrome in two pediatric populations: Implications for genetic screening of deaf and hard of hearing children. Genet Med. 2010;12(8):512-6.

4. Mathur P, Yang J. Usher syndrome: Hearing loss, retinal degeneration and associated abnormalities. Biochim Biophys Acta. 2015;1852(3):406-20.

5. Yan D, Liu XZ. Genetics and pathological mechanisms of Usher syndrome. J Hum Genet. 2010;55(6):327-35.
6. Boughman JA, Vernon M, Shaver KA. Usher syndrome: definition and estimate of prevalence from two high-risk populations. J Chronic Dis. 1983; 36(8):595-603.

7. Slijkerman RW, Vache C, Dona M, Garcia-Garcia G, Claustres M, Hetterschijt L, Peters TA, Hartel BP, Pennings RJ, Millan JM, et al. Antisense Oligonucleotide-based Splice Correction for USH2Aassociated Retinal Degeneration Caused by a Frequent Deep-intronic Mutation. Mol Ther Nucleic Acids. 2016;5(10):e381.

8. Trouillet A, Dubus E, Degardin J, Estivalet A, Ivkovic I, Godefroy D, GarciaAyuso D, Simonutti M, Sahly I, Sahel JA, et al. Cone degeneration is triggered by the absence of USH1 proteins but prevented by antioxidant treatments. Sci Rep. 2018;8(1):1968.

9. Donato L, Scimone C, Nicocia G, Denaro L, Robledo R, Sidoti A, D'Angelo R. GLO1 gene polymorphisms and their association with retinitis pigmentosa: a case-control study in a Sicilian population. Mol Biol Rep. 2018;45(5):1349-55.

10. Lewis MA, Nolan LS, Cadge BA, Matthews LJ, Schulte BA, Dubno JR, Steel $\mathrm{KP}$, Dawson SJ. Whole exome sequencing in adult-onset hearing loss reveals a high load of predicted pathogenic variants in known deafnessassociated genes and identifies new candidate genes. BMC Med Genomics. 2018;11(1):77.

11. Sakuma N, Moteki H, Takahashi M, Nishio SY, Arai Y, Yamashita Y, Oridate N, Usami S. An effective screening strategy for deafness in combination with a next-generation sequencing platform: a consecutive analysis. J Hum Genet. 2016;61(3):253-61.

12. Taylor KR, Deluca AP, Shearer AE, Hildebrand MS, Black-Ziegelbein EA, Anand VN, Sloan CM, Eppsteiner RW, Scheetz TE, Huygen PL, et al. AudioGene: predicting hearing loss genotypes from phenotypes to guide genetic screening. Hum Mutat. 2013;34(4):539-45.

13. Abecasis GR, Cherny SS, Cookson WO, Cardon LR. Merlin--rapid analysis of dense genetic maps using sparse gene flow trees. Nat Genet. 2002;30(1):97101.

14. Hereditary Hearing Loss Homepage [http://hereditaryhearingloss.org].

15. Mazzoli MGVC, Newton V, Giarbini N, Declau F, Parving A Recommendations for the description of genetic and audiological data for families with nonsyndromic hereditary hearing impairment. Audiological Medicine. 2003;1(2):148-50.

16. Richards S, Aziz N, Bale S, Bick D, Das S, Gastier-Foster J, Grody WW, Hegde

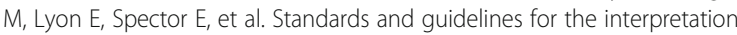
of sequence variants: a joint consensus recommendation of the American College of Medical Genetics and Genomics and the Association for Molecular Pathology. Genet Med. 2015;17(5):405-24.

17. Lentz J, Keats B. Usher Syndrome Type II. In: Adam MP, Ardinger HH, Pagon RA, Wallace SE, Bean LH, Stephens K, Amemiya A, editors. GeneReviews((R)). Seattle (WA): 1993

18. Lenassi E, Vincent A, Li Z, Saihan Z, Coffey AJ, Steele-Stallard HB, Moore AT,

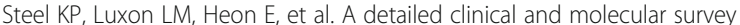
of subjects with nonsyndromic USH2A retinopathy reveals an allelic hierarchy of disease-causing variants. European journal of human genetics: EJHG. 2015;23(10):1318-27.

19. Abadie C, Blanchet C, Baux D, Larrieu L, Besnard T, Ravel P, Biboulet R, Hamel C, Malcolm S, Mondain M, et al. Audiological findings in 100 USH2 patients. Clinical genetics. 2012;82(5):433-8.

20. Fuster-Garcia C, Garcia-Garcia G, Jaijo T, Fornes N, Ayuso C, FernandezBurriel M. Sanchez-De la Morena A, Aller E, Millan JM: High-throughput sequencing for the molecular diagnosis of Usher syndrome reveals 42 novel mutations and consolidates CEP250 as Usher-like disease causative. Sci Rep. 2018;8(1):17113.

21. Schwartz SB, Aleman TS, Cideciyan AV, Windsor EA, Sumaroka A, Roman AJ, Rane T, Smilko EE, Bennett J, Stone EM, et al. Disease expression in Usher syndrome caused by VLGR1 gene mutation (USH2C) and comparison with USH2A phenotype. Invest Ophthalmol Vis Sci. 2005;46(2):734-43.

22. Besnard T, Vache C, Baux D, Larrieu L, Abadie C, Blanchet C, Odent S, Blanchet $\mathrm{P}$, Calvas $\mathrm{P}$, Hamel $\mathrm{C}$, et al. Non-USH2A mutations in USH2 patients. Hum Mutat. 2012;33(3):504-10.

23. Nakayama J, Fu YH, Clark AM, Nakahara S, Hamano K, Iwasaki N, Matsui A, Arinami T, Ptacek $L$ J. A nonsense mutation of the MASS1 gene in a family with febrile and afebrile seizures. Ann Neurol. 2002;52(5):654-7.

24. Skradski SL, Clark AM, Jiang H, White HS, Fu YH, Ptacek LJ. A novel gene causing a mendelian audiogenic mouse epilepsy. Neuron. 2001;31(4):537-44. 
25. Matthijs G, Souche E, Alders M, Corveleyn A, Eck S, Feenstra I, Race V, Sistermans E, Sturm M, Weiss M, et al. Guidelines for diagnostic nextgeneration sequencing. European journal of human genetics: EJHG. 2016; 24(10):1515.

26. GeneReviews: Usher Syndrome Type II [https://www.ncbi.nlm.nih.gov/ books/NBK1341/].

27. Eandi CM, Dallorto L, Spinetta R, Micieli MP, Vanzetti M, Mariottini A, Passerini I, Torricelli F, Alovisi C, Marchese C. Targeted next generation sequencing in Italian patients with Usher syndrome: phenotype-genotype correlations. Sci Rep. 2017;7(1):15681.

28. Garcia-Garcia G, Besnard T, Baux D, Vache C, Aller E, Malcolm S, Claustres M, Millan JM, Roux AF. The contribution of GPR98 and DFNB31 genes to a Spanish Usher syndrome type 2 cohort. Mol Vis. 2013;19:367-73.

29. Liu X, Bulgakov OV, Darrow KN, Pawlyk B, Adamian M, Liberman MC, Li T. Usherin is required for maintenance of retinal photoreceptors and normal development of cochlear hair cells. Proc Natl Acad Sci U S A. 2007;104(11): $4413-8$.

30. Richardson GP, Petit C. Hair-Bundle Links: Genetics as the Gateway to Function. Cold Spring Harb Perspect Med. 2019.

31. Yang J, Liu X, Zhao Y, Adamian M, Pawlyk B, Sun X, McMillan DR, Liberman MC, Li T. Ablation of whirlin long isoform disrupts the USH2 protein complex and causes vision and hearing loss. PLoS Genet. 2010;6(5): e1000955.

32. Jouret G, Poirsier C, Spodenkiewicz M, Jaquin C, Gouy E, Arndt C, Labrousse M, Gaillard D, Doco-Fenzy M, Lebre AS. Genetics of Usher Syndrome: New Insights From a Meta-analysis. Otol Neurotol. 2019;40(1):121-9.

Ready to submit your research? Choose BMC and benefit from:

- fast, convenient online submission

- thorough peer review by experienced researchers in your field

- rapid publication on acceptance

- support for research data, including large and complex data types

- gold Open Access which fosters wider collaboration and increased citations

- maximum visibility for your research: over $100 \mathrm{M}$ website views per year

At BMC, research is always in progress.

Learn more biomedcentral.com/submissions 